This is a self-archived version of an original article. This version may differ from the original in pagination and typographic details.

Author(s): Siltakoski, Jarkko

Title: Equivalence of viscosity and weak solutions for the normalized $p(x)$-Laplacian

Year: 2018

Version: Accepted version (Final draft)

Copyright: @ Springer-Verlag GmbH Germany, part of Springer Nature 2018.

Rights: In Copyright

Rights url: http://rightsstatements.org/page/lnC/1.0/?language=en

Please cite the original version:

Siltakoski, J. (2018). Equivalence of viscosity and weak solutions for the normalized $p(x)$ Laplacian. Calculus of Variations and Partial Differential Equations, 57(4), Article 95.

https://doi.org/10.1007/s00526-018-1375-1 


\title{
EQUIVALENCE OF VISCOSITY AND WEAK SOLUTIONS FOR THE NORMALIZED $p(x)$-LAPLACIAN
}

\author{
JARKKO SILTAKOSKI
}

\begin{abstract}
We show that viscosity solutions to the normalized $p(x)$-Laplace equation coincide with distributional weak solutions to the strong $p(x)$-Laplace equation when $p$ is Lipschitz and $\inf p>1$. This yields $C^{1, \alpha}$ regularity for the viscosity solutions of the normalized $p(x)$-Laplace equation. As an additional application, we prove a Radó-type removability theorem.
\end{abstract}

\section{INTRODUCTION}

In this paper, we study viscosity solutions to the normalized $p(x)$-Laplace equation which is defined by

$$
-\Delta_{p(x)}^{N} u:=-\Delta u-\frac{p(x)-2}{|D u|^{2}} \Delta_{\infty} u=0
$$

where

$$
\Delta_{\infty} u:=\left\langle D^{2} u D u, D u\right\rangle .
$$

There has been recent interest in normalized equations, see e.g. [JS17, IJS, BG15]. We are partly motivated by the connection to stochastic tug-of-war games [PS08, PSSW09] as the case of space dependent probabilities leads to (1.1) [AHP17].

The objective of this work is to show that viscosity solutions to (1.1) coincide with solutions in the distributional weak sense, when the equation is rewritten in an appropriate divergence formulation. One approach to this kind of equivalence results [JLM01, Ish95] is based on the uniqueness of solutions. However, it seems difficult to use uniqueness in our case because the uniqueness of solutions is an open problem for the equation (1.1) as pointed out in [JLP10]. The equation (1.1) is in non-divergence form. In order to find the appropriate weak formulation, we note that for $u \in C^{2}(\Omega)$ with non-vanishing gradient it holds that

$$
-|D u|^{p(x)-2} \Delta_{p(x)}^{N} u=-\operatorname{div}\left(|D u|^{p(x)-2} D u\right)+|D u|^{p(x)-2} \log (|D u|) D u \cdot D p .
$$

Thus the weak formulation of (1.1) should be the strong $p(x)$-Laplace equation

$$
-\Delta_{p(x)}^{S} u:=-\operatorname{div}\left(|D u|^{p(x)-2} D u\right)+|D u|^{p(x)-2} \log |D u| D u \cdot D p=0 .
$$

Our main result, Theorem 5.9, is that viscosity solutions to (1.1) coincide with weak solutions to (1.2) when the function $p$ is Lipschitz with inf $p>1$. With these assumptions weak solutions to (1.2) in a domain are locally $C^{1, \alpha}$ continuous [ZZ12]. Thus our equivalence result yields local $C^{1, \alpha}$ regularity also for viscosity solutions to (1.1). As an application, we prove a Radó-type removability theorem for the strong $p(x)$-Laplacian. The theorem follows from the equivalence result since in the definition of a viscosity solution we may ignore the test functions whose gradient vanishes. The equivalence result also implies that the equation (1.2) is homogeneous: if $u$ is a solution, so is $\lambda u$. This is not completely obvious and was established in [AH10].

That viscosity solutions to (1.1) are weak solutions to (1.2) is proven by applying the method of [JJ12]. The idea is to approximate a viscosity solution through a 
sequence of inf-convolutions, show that the inf-convolutions are essentially weak supersolutions, and then pass to the limit.

First, in Lemma 5.3 we show that the inf-convolution $u_{\varepsilon}$ of a viscosity supersolution $u$ to (1.1) is still, in essence, a viscosity supersolution up to some error. This fact is a key part of our proof. If there was no $x$-dependence in (1.1), it would be straightforward to see that the inf-convolution of a viscosity supersolution is still a viscosity supersolution. This is because a test function that touches the inf-convolution from below also touches the original function from below at a nearby point once we add some constant to it. From this it would follow that the inf-convolution is a supersolution to the original equation. However, the equation (1.1) has $x$-dependence caused by $p(x)$. Thus the inf-convolution no longer satisfies the original equation.

In Lemma 5.5 we use the standard mollification on $u_{\varepsilon}$ and $p$ to deduce from Lemma 5.3 that $u_{\varepsilon}$ is "almost" a weak solution to $-\Delta_{p(x)}^{S} u_{\varepsilon} \geq 0$. Applying Caccioppoli type estimates and vector inequalities we are then able to deduce that the sequence of inf-convolutions converges to the viscosity supersolution in $W_{l o c}^{1, p(\cdot)}(\Omega)$ as $\varepsilon \rightarrow 0$. This allows us to pass to the limit and conclude that the function $u$ satisfies $-\Delta_{p(x)}^{S} u \geq 0$ in the weak sense.

Due to the variable exponent, the operator $\Delta_{p(x)}^{S}$ can be singular in some subsets and degenerate in others. Therefore we apply different arguments in the cases $p(x)<2$ and $p(x) \geq 2$, and finally need to be able to combine them.

The equivalence of weak and viscosity solutions to the usual $p$-Laplace equation was first proven by Juutinen, Lindqvist and Manfredi [JLM01]. Later Julin and Juutinen [JJ12] presented a more direct way to show that viscosity solutions to $-\Delta_{p} u=f$ are also weak solutions. This proof was adapted in [APR17] to show that viscosity solutions to $-\Delta_{p}^{N} u=f$ coincide with weak solutions to $-\Delta_{p} u=$ $|D u|^{p-2} f$ when $p \geq 2$. Similar arguments were also used in [MO] to study the equivalence of solutions to $-\Delta_{p} u=f(x, u, D u)$. The variable exponent case was explored in [JLP10] where the equivalence of weak and viscosity solutions was proven for the $p(x)$-Laplace equation using techniques of [JLM01].

As mentioned, the equation (1.1) appears in stochastic tug-of-war games. Let us illustrate this in the case where $p>2$ is a constant by considering the following two-player, zero-sum game from [MPR12]. A step size $\varepsilon>0$ is fixed and a token is placed at $x_{0}$ in a domain $\Omega$. The players toss a biased coin that is heads with the probability $\alpha=\frac{p-2}{p+N}$ and tails with the probability $\beta=1-\alpha$. If the outcome is heads, the following tug-of-war step is played: a fair coin is tossed and the winning player is allowed to move the token to any position $x_{1} \in B_{\varepsilon}\left(x_{0}\right)$. If the outcome is tails, the token moves to a random position in $x_{1} \in B_{\varepsilon}\left(x_{0}\right)$. Once the token exits the domain, the game ends and player I pays player II according to the final location of the token. When the players optimize over their strategies, we obtain a value of the game. Then, as the step-size approaches zero, the value function converges uniformly to a viscosity solution of $-\Delta_{p}^{N} u=0$ in $\Omega$. This result can be extended to the general case $1<p(x)<\infty$, see [PS08, AHP17].

The equation (1.2) was introduced by Adamowicz and Hästö [AH10] in connection with mappings of finite distortion. Unlike the standard $p(x)$-Laplace equation, the equation (1.2) is homogeneous and its solutions satisfy a classical Harnack inequality [AH11]. The equation (1.2) has been further studied for example in [ZZ12, PL13, ZZZ17].

The paper is organized as follows: in Section 2 we recall the variable exponent Lebesgue and Sobolev spaces. Section 3 contains the rigorous definitions of solutions to equations (1.1) and (1.2). In Section 4 we show that weak solutions of (1.2) are viscosity solutions to (1.1) and the converse statement is proven in 
Section 5. Finally, in Section 6 we formulate and prove a Radó-type removability theorem for weak solutions of (1.2).

\section{Variable EXPonent Lebesgue and SOBoleV SPACES}

We briefly recall basic facts about these spaces. For general reference see e.g. [DHHR11]. Let $\Omega \subset \mathbb{R}^{N}$ be an open and bounded set and let $p: \Omega \rightarrow(1, \infty)$ be a measurable function. We denote

$$
p_{\max }:=\operatorname{ess~sup}_{x \in \Omega} p(x) \text { and } p_{\min }:=\operatorname{essinf}_{x \in \Omega} p(x) .
$$

The variable exponent Lebesgue space $L^{p(\cdot)}(\Omega)$ is defined as the set of measurable functions $u: \Omega \rightarrow \mathbb{R}$ for which the $p(\cdot)$-modular

$$
\varrho_{p(\cdot)}(u):=\int_{\Omega}|u|^{p(x)} d x
$$

is finite. It is a Banach space equipped with the Luxemburg norm

$$
\|u\|_{L^{p(\cdot)(\Omega)}}:=\inf \left\{\lambda>0: \int_{\Omega}\left|\frac{u}{\lambda}\right|^{p(x)} d x \leq 1\right\} .
$$

Given that $p_{\max }<\infty$ or $\varrho_{p(\cdot)}(u)>0$, the norm and the modular satisfy the inequality (see [DHHR11, p75])

$$
\begin{aligned}
\min \left\{\varrho_{p(\cdot)}(u)^{\frac{1}{p_{\min }}}, \varrho_{p(\cdot)}(u)^{\frac{1}{p_{\max }}}\right\} & \leq\|u\|_{L^{p(\cdot)}(\Omega)} \\
& \leq \max \left\{\varrho_{p(\cdot)}(u)^{\frac{1}{p_{\min }}}, \varrho_{p(\cdot)}(u)^{\frac{1}{p_{\max }}}\right\} .
\end{aligned}
$$

A version of Hölder's inequality holds [DHHR11, p81] : if $u \in L^{p(\cdot)}(\Omega)$ and $v \in L^{p^{\prime}(\cdot)}(\Omega)$, where $\frac{1}{p(x)}+\frac{1}{p^{\prime}(x)}=1$ for a.e. $x \in \Omega$, then

$$
\int_{\Omega}|u||v| d x \leq 2\|u\|_{L^{p(\cdot)}(\Omega)}\|v\|_{L^{p^{\prime}(\cdot)}(\Omega)} .
$$

As a consequence of the Hölder's inequality we have that

$$
\|u\|_{L^{q(\cdot)}(\Omega)} \leq 2(1+|\Omega|)\|u\|_{L^{p(\cdot)}(\Omega)}
$$

for all $u \in L^{p(\cdot)}(\Omega)$ if $q(x) \leq p(x)$ for a.e. $x \in \Omega$.

If $1<p_{\min } \leq p_{\max }<\infty$, then $L^{p(\cdot)}(\Omega)$ is reflexive and the dual of $L^{p(\cdot)}(\Omega)$ is $L^{p^{\prime}(\cdot)}(\Omega)$.

The variable exponent Sobolev space $W^{1, p(\cdot)}(\Omega)$ is the set of functions in $u \in$ $L^{p(\cdot)}(\Omega)$ for which the weak gradient $D u$ belongs in $L^{p(\cdot)}(\Omega)$. It is a Banach space equipped with the norm

$$
\|u\|_{W^{1, p(\cdot)}(\Omega)}:=\|u\|_{L^{p(\cdot)}(\Omega)}+\|D u\|_{L^{p(\cdot)}(\Omega)} .
$$

The space $W_{0}^{1, p}(\Omega)$ is the closure of compactly supported Sobolev functions in the space $W^{1, p(\cdot)}(\Omega)$. A function belongs to the the local Lebesgue space $L_{l o c}^{p(\cdot)}(\Omega)$ if it belongs to $L^{p(\cdot)}\left(\Omega^{\prime}\right)$ for all $\Omega^{\prime} \Subset \Omega$. The space $W_{l o c}^{1, p(\cdot)}(\Omega)$ is defined analogically.

\section{The Strong and normalized $p(x)$-Laplace Equations}

In this section, we define weak solutions to the strong $p(x)$-Laplace equation and viscosity solutions to the normalized $p(x)$-Laplace equation.

From now on we assume that $p$ is Lipschitz continuous and $p_{\min }>1$. 
Definition 3.1. A function $u \in W_{l o c}^{1, p(\cdot)}(\Omega)$ is a weak supersolution to $-\Delta_{p(x)}^{S} u \geq 0$ in $\Omega$ if

$$
\int_{\Omega}|D u|^{p(x)-2} D u \cdot D \varphi+|D u|^{p(x)-2} \log (|D u|) D u \cdot D p \varphi d x \geq 0
$$

for all non-negative $\varphi \in W^{1, p(\cdot)}(\Omega)$ with compact support. We say that $u$ is a weak subsolution to $-\Delta_{p(x)}^{S} u \leq 0$ if $-u$ is a supersolution and that $u$ is a weak solution to $-\Delta_{p(x)}^{S} u=0$ if $u$ is both supersolution and subsolution.

Lemma 3.2. It is enough to consider $C_{0}^{\infty}(\Omega)$ test functions in the previous definition.

Proof. Assume that $\varphi \in W^{1, p(\cdot)}(\Omega)$ has a compact support in an open set $\Omega^{\prime} \Subset \Omega$. Since $p$ is log-Hölder continuous and bounded as a Lipschitz function, there is a sequence of functions $\varphi_{j} \in C_{0}^{\infty}\left(\Omega^{\prime}\right)$ such that $\varphi_{j} \rightarrow \varphi$ in $W^{1, p(\cdot)}\left(\Omega^{\prime}\right)$ (see [DHHR11, p347]). We set $\psi_{j}:=\varphi-\varphi_{j}$. Then it is enough to show that

$$
\int_{\Omega^{\prime}}|D u|^{p(x)-2} D u \cdot D \psi_{j} d x+\int_{\Omega^{\prime}}|D u|^{p(x)-2} \log (|D u|) D u \cdot D p \psi_{j} d x \rightarrow 0
$$

as $j \rightarrow \infty$. The first integral convergences to zero by Hölder's inequality so we focus on the second integral. We may assume that $N>1$. We set $q(x):=\frac{p(x)}{p(x)-1+\frac{1}{N}}$. Using the inequality $a^{s} \log a \leq N a^{s+\frac{1}{N}}+\frac{1}{s}$ for $a, s>0$ we get

$$
\begin{aligned}
& \int_{\Omega^{\prime}}|D u|^{p(x)-1}|\log | D u|||D p|\left|\psi_{j}\right| d x \\
& \quad \leq\|D p\|_{L^{\infty}\left(\Omega^{\prime}\right)}\left(\int_{\Omega^{\prime}} \frac{\left|\psi_{j}\right|}{p(x)-1} d x+N \int_{\Omega^{\prime}}|D u|^{p(x)-1+\frac{1}{N}}\left|\psi_{j}\right| d x\right) \\
& \quad \leq C(p, \Omega)\left(\left\|\psi_{j}\right\|_{L^{p(\cdot)\left(\Omega^{\prime}\right)}}+\left\||D u|^{p(x)-1+\frac{1}{N}}\right\|_{L^{q(\cdot)\left(\Omega^{\prime}\right)}}\left\|\psi_{j}\right\|_{L^{q^{(\cdot)}\left(\Omega^{\prime}\right)}}\right) .
\end{aligned}
$$

We take $r \in(1, N)$ such that $q^{+} \leq r^{*}:=\frac{N r}{N-r}$. Then we have $q^{\prime}(x)=\frac{N p(x)}{N-1} \leq$ $\min \left(p^{*}(x), r^{*}\right)$, where $p^{*}(x):=\frac{N p(x)}{N-p(x)}$. Therefore

$$
\left\|\psi_{j}\right\|_{L^{q^{\prime}(\cdot)\left(\Omega^{\prime}\right)}} \leq 2(1+|\Omega|)\left\|\psi_{j}\right\|_{L^{\min \left(p^{*}(\cdot), r^{*}\right)}\left(\Omega^{\prime}\right)} .
$$

Since $\psi_{j} \in W_{0}^{1, \min (p(\cdot), r)}\left(\Omega^{\prime}\right)$, we have by a variable exponent version of the Sobolev inequality (see e.g. [DHHR11, p265])

$$
\left\|\psi_{j}\right\|_{L^{\min \left(p^{*}(\cdot), r^{*}\right)}\left(\Omega^{\prime}\right)} \leq C\left\|D \psi_{j}\right\|_{L^{\min (p(\cdot), r)}\left(\Omega^{\prime}\right)} \leq 2 C(1+|\Omega|)\left\|D \psi_{j}\right\|_{L^{p(\cdot)}\left(\Omega^{\prime}\right)} .
$$

These estimates imply the claim since $\left\|\psi_{j}\right\|_{W^{1, p}\left(\Omega^{\prime}\right)} \rightarrow 0$ as $j \rightarrow \infty$.

In order to define viscosity solutions to $-\Delta_{p(x)}^{N} u=0$, we set

$$
F(x, \eta, X):=-\left(\operatorname{tr} X+\frac{p(x)-2}{|\eta|^{2}}\langle X \eta, \eta\rangle\right)
$$

for all $(x, \eta, X) \in \Omega \times\left(\mathbb{R}^{N} \backslash\{0\}\right) \times S^{N}$ where $S^{N}$ is the set of symmetric $N \times N$ matrices. We also recall the concept of semi-jets. The subjet of a function $u: \Omega \rightarrow$ $\mathbb{R}$ at $x$ is defined by setting $(\eta, X) \in J^{2,-} u(x)$ if

$$
u(y) \geq u(x)+\eta \cdot(y-x)+\frac{1}{2}\langle X(y-x),(y-x)\rangle+o\left(|y-x|^{2}\right) \text { as } y \rightarrow x .
$$

The closure of a subjet is defined by setting $(\eta, X) \in \bar{J}^{2,-} u(x)$ if there is a sequence $\left(\eta_{i}, X_{i}\right) \in J^{2,-} u\left(x_{i}\right)$ such that $\left(x_{i}, \eta_{i}, X_{i}\right) \rightarrow(x, \eta, X)$. The superjet $J^{2,+} u(x)$ and its closure $\bar{J}^{2,+} u(x)$ are defined in the same manner except that the inequality (3.1) is reversed. 
Definition 3.3. A lower semicontinuous function $u: \Omega \rightarrow \mathbb{R}$ is a viscosity supersolution to $-\Delta_{p(x)}^{N} u \geq 0$ in $\Omega$ if, whenever $(\eta, X) \in J^{2,-} u(x)$ with $x \in \Omega$ and $\eta \neq 0$, then

$$
F(x, \eta, X) \geq 0 .
$$

A function $u$ is a viscosity subsolution to $-\Delta_{p(x)}^{N} u \leq 0$ if $-u$ is a viscosity supersolution, and a viscosity solution to $-\Delta_{p(x)}^{N} u=0$ if it is both viscosity super- and subsolution.

Remark. Observe that in the previous definition we require nothing in the case $(0, X) \in J^{2,-} u(x)$.

Viscosity solutions may be equivalently defined using the jet-closures or test functions. The next proposition follows easily from the proof of Proposition 2.6 in [Koi12].

Proposition 3.4. Let $u: \Omega \rightarrow \mathbb{R}$ be lower semicontinuous. Then the following conditions are equivalent.

(i) The function $u$ is a viscosity supersolution to $-\Delta_{p(x)}^{N} u \geq 0$ in $\Omega$.

(ii) Whenever $(\eta, X) \in \bar{J}^{2,-} u(x)$ with $x \in \Omega, \eta \neq 0$, we have $F(x, \eta, X) \geq 0$.

(iii) Whenever $\varphi \in C^{2}(\Omega)$ is such that $\varphi(x)=u(x), D \varphi(x) \neq 0$ and $\varphi(y)<$ $u(y)$ for all $y \neq x$, it holds $F\left(x, D \varphi(x), D^{2} \varphi(x)\right) \geq 0$.

When $\varphi$ is as in the third condition above, we say that $\varphi$ touches $u$ from below at $x$.

\section{WEAK SOLUTiOns ARE Viscosity SOLUTiOns}

We show that if $u$ is a weak solution to $-\Delta_{p(x)}^{S} u=0$, then it is a viscosity solution to $-\Delta_{p(x)}^{N} u=0$.

Juutinen, Lukkari and Parviainen [JLP10] showed that weak solutions to the standard $p(x)$-Laplace equation are also viscosity solutions. This was accomplished with the help of the comparison principle. For if $u$ is a weak supersolution to $-\Delta_{p(x)} u \geq 0$ that is not a viscosity supersolution, then there is a test function $\varphi \in C^{2}$ touching $u$ from below at $x$ so that $-\Delta_{p(x)} \varphi<0$ in some ball $B(x)$. Lifting $\varphi$ slightly produces a new function $\tilde{\varphi}$ still satisfying $-\Delta_{p(x)} \tilde{\varphi}<0$ in $B(x)$ and $\tilde{\varphi} \leq u$ in $\partial B(x)$. Comparison principle now implies that $\tilde{\varphi} \leq u$ in $B(x)$ which is a contradiction since $\tilde{\varphi}(x)>\varphi(x)=u(x)$.

Our difficulty is that, to the best of our knowledge, the comparison principle is an open problem for the strong $p(x)$-Laplacian. Our strategy is therefore to consider a ball so small that the gradient of the test function does not vanish. Then the comparison principle holds and we arrive at a contradiction.

Theorem 4.1. If $u \in W_{l o c}^{1, p(\cdot)}(\Omega)$ is a weak solution to $-\Delta_{p(x)}^{S} u=0$, then it is a viscosity solution to $-\Delta_{p(x)}^{N} u=0$ in $\Omega$.

Proof. Zhang and Zhou [ZZ12] showed that weak solutions of $-\Delta_{p(x)}^{S} u=0$ are in $C^{1}(\Omega)$. Therefore it suffices to show that if $u \in C^{1}(\Omega)$ is a weak supersolution to $-\Delta_{p(x)}^{S} u \geq 0$, then it is also a viscosity supersolution to $-\Delta_{p(x)}^{N} u \geq 0$. Assume on the contrary that there is $\varphi \in C^{2}(\Omega)$ touching $u$ from below at $x_{0} \in \Omega, D \varphi\left(x_{0}\right) \neq 0$ and

$$
0>-h>F\left(x_{0}, D \varphi\left(x_{0}\right), D^{2} \varphi\left(x_{0}\right)\right) .
$$

Then by continuity there is $r>0$ such that in $B_{r}\left(x_{0}\right)$ it holds

$$
-h|D \varphi|^{p(x)-2} \geq-|D \varphi|^{p(x)-2}\left(\Delta \varphi+\frac{p(x)-2}{|D \varphi|^{2}} \Delta_{\infty} \varphi\right) .
$$


Since $D u\left(x_{0}\right)=D \varphi\left(x_{0}\right) \neq 0$, we may also assume that there is $m>0$ such that

$$
\inf _{x \in B_{r}\left(x_{0}\right)}|D \varphi|^{p(x)-2} \geq m
$$

and

$$
\underset{x \in B_{r}\left(x_{0}\right)}{\left.\operatorname{ess} \sup _{0}|D p||| D \varphi\right|^{p(x)-2} \log (|D \varphi|) D \varphi-|D u|^{p(x)-2} \log (|D u|) D u \mid \leq \frac{h m}{2} .}
$$

Let $l:=\min _{x \in \partial B_{r}\left(x_{0}\right)}(u-\varphi)>0$ and set $\psi(x):=\max (\varphi(x)+l-u(x), 0)$. Then $\psi \in W_{0}^{1,2}\left(B_{r}\left(x_{0}\right)\right)$ so there are $\psi_{j} \in C_{0}^{\infty}\left(B_{r}\left(x_{0}\right)\right)$ such that $\psi_{j} \rightarrow \psi$ in $W^{1,2}\left(B_{r}\left(x_{0}\right)\right)$. Let $p_{j}$ be the standard mollification of $p$. Multiplying (4.1) by $\psi$ and integrating over $B_{r}\left(x_{0}\right)$ yields

$$
\begin{aligned}
-h \int_{B_{r}\left(x_{0}\right)} & |D \varphi|^{p(x)-2} \psi d x \\
\quad \geq & \int_{B_{r}\left(x_{0}\right)}-|D \varphi|^{p(x)-2}\left(\Delta \varphi+\frac{p(x)-2}{|D \varphi|^{2}} \Delta_{\infty} \varphi\right) \psi d x \\
\quad= & \lim _{j \rightarrow \infty} \int_{B_{r}\left(x_{0}\right)}-|D \varphi|^{p_{j}(x)-2}\left(\Delta \varphi+\frac{p_{j}(x)-2}{|D \varphi|^{2}} \Delta_{\infty} \varphi\right) \psi_{j} d x
\end{aligned}
$$

where the last equality holds because $\psi_{j} \rightarrow \psi$ in $W^{1,2}\left(B_{r}\left(x_{0}\right)\right)$ and $p_{j} \rightarrow p$ uniformly in $B_{r}\left(x_{0}\right)$. Calculating the divergence of $|D \varphi|^{p_{j}(x)-2} D \varphi$ and integrating by parts we get

$$
\begin{aligned}
\int_{B_{r}\left(x_{0}\right)} & -|D \varphi|^{p_{j}(x)-2}\left(\Delta \varphi+\frac{p_{j}(x)-2}{|D \varphi|^{2}} \Delta_{\infty} \varphi\right) \psi_{j} d x \\
= & \int_{B_{r}\left(x_{0}\right)}-\operatorname{div}\left(|D \varphi|^{p_{j}(x)-2} D \varphi\right) \psi_{j}+|D \varphi|^{p_{j}(x)-2} \log (|D \varphi|) D \varphi \cdot D p_{j} \psi_{j} d x \\
= & \int_{B_{r}\left(x_{0}\right)}|D \varphi|^{p_{j}(x)-2} D \varphi \cdot\left(D \psi_{j}+\log (|D \varphi|) D p_{j} \psi_{j}\right) d x
\end{aligned}
$$

By the convergence of $\psi_{j}$ and $p_{j}$, it follows from (4.4) and (4.5) that

$$
-h \int_{B_{r}\left(x_{0}\right)}|D \varphi|^{p(x)-2} \psi d x \geq \int_{B_{r}\left(x_{0}\right)}|D \varphi|^{p(x)-2} D \varphi \cdot(D \psi+\log (|D \varphi|) D p \psi) d x .
$$

Since $u$ is a weak supersolution to $\Delta_{p(x)}^{S} u=0$ and $\psi \in W^{1, p(\cdot)}(\Omega)$ has a compact support in $\Omega$, we have

$$
\int_{B_{r}\left(x_{0}\right)}|D u|^{p(x)-2} D u \cdot(D \psi+\log |D u| D p \psi) d x \geq 0 .
$$

Denoting $A:=\left\{x \in B_{r}\left(x_{0}\right): \psi(x)>0\right\}$ and combining (4.6) and (4.7) we arrive at

$$
\begin{aligned}
& \int_{A}\left(|D \varphi|^{p(x)-2} D \varphi-|D u|^{p(x)-2} D u\right) \cdot(D \varphi-D u) d x \\
& \leq\left.\int_{A}|| D u\right|^{p(x)-2} \log (|D u|) D u-|D \varphi|^{p(x)-2} \log (|D \varphi|) D \varphi|| D p \mid \psi d x \\
& \quad-h \int_{A}|D \varphi|^{p(x)-2} \psi d x \\
& \leq-\frac{h m}{2} \int_{A} \psi d x
\end{aligned}
$$


where the last inequality follows from (4.2) and (4.3). Since

$$
\left(|a|^{p(x)-2} a-|b|^{p(x)-2} b\right) \cdot(a-b) \geq 0
$$

for any two vectors $a, b \in \mathbb{R}^{N}$ when $p(x)>1$, it follows from (4.8) that $|A|=0$. But this is impossible since $\varphi\left(x_{0}\right)=u\left(x_{0}\right)$ and $l>0$.

\section{Viscosity solutions are Weak solutions}

We show that if $u$ is a viscosity supersolution to $-\Delta_{p(x)}^{N} u \geq 0$, then it is a weak supersolution to $-\Delta_{p(x)}^{S} u \geq 0$. The same statement for subsolutions then follows by analogy.

We recall the usual partial ordering for symmetric $N \times N$ matrices by setting $X \leq Y$ if $\langle X \xi, \xi\rangle \leq\langle Y \xi, \xi\rangle$ for all $\xi \in \mathbb{R}^{N}$. For a matrix $X$ we also set $\|X\|:=$ $\max \{|\lambda|: \lambda$ is an eigenvalue of $X\}$ and for vectors $\xi, \eta \in \mathbb{R}^{N}$ we use the notation $\xi \otimes \eta:=\xi \eta^{\prime}$, i.e. $\xi \otimes \eta$ is an $N \times N$ matrix whose $(i, j)$ entry is $\xi_{i} \eta_{j}$.

Definition 5.1 (Inf-convolution). Let $q \geq 2$ and $\varepsilon>0$. The inf-convolution of a bounded function $u \in C(\Omega)$ is defined by

$$
u_{\varepsilon}(x):=\inf _{y \in \Omega}\left\{u(y)+\frac{1}{q \varepsilon^{q-1}}|x-y|^{q}\right\} .
$$

The inf-convolution is well known to provide good approximations of viscosity supersolutions and often one only needs to consider it for $q=2$ (see e.g. [CIL92]). However, as the authors in [JJ12] observed, considering large enough $q$ essentially cancels the singularity in the usual $p$-Laplace operator when $1<p<2$. In similar fashion it also cancels the singularity of the operator $\Delta_{p(x)}^{S}$. This is due to the property (v) in the next lemma. We also list some other properties of the infconvolution.

Lemma 5.2. Let $u \in C(\Omega)$ be a bounded function. Then the inf-convolution $u_{\varepsilon}$ as defined in (5.1) has the following properties.

(i) We have $u_{\varepsilon} \leq u$ in $\Omega$ and $u_{\varepsilon} \rightarrow u$ locally uniformly in $\Omega$ as $\varepsilon \rightarrow 0$.

(ii) There exists $r(\varepsilon)>0$ such that

$$
u_{\varepsilon}(x)=\inf _{y \in B_{r(\varepsilon)}(x) \cap \Omega}\left\{u(y)+\frac{1}{q \varepsilon^{q-1}}|x-y|^{q}\right\}
$$

and $r(\varepsilon) \rightarrow 0$ as $\varepsilon \rightarrow 0$. In fact we can choose $r(\varepsilon)=\left(q \varepsilon^{q-1} \operatorname{osc}_{\Omega} u\right)^{\frac{1}{q}}$.

(iii) The function $u_{\varepsilon}$ is semi-concave in $\Omega_{r(\varepsilon)}$, that is, the function $x \mapsto u_{\varepsilon}(x)-$ $\frac{q-1}{2 \varepsilon^{q-1}} r(\varepsilon)^{q-2}|x|^{2}$ is concave.

(iv) If $x \in \Omega_{r(\varepsilon)}:=\{x \in \Omega: \operatorname{dist}(x, \partial \Omega)>r(\varepsilon)\}$, then there exists a point $x_{\varepsilon} \in$ $B_{r(\varepsilon)}(x)$ such that $u_{\varepsilon}(x)=u\left(x_{\varepsilon}\right)+\frac{1}{q \varepsilon^{q-1}}\left|x-x_{\varepsilon}\right|^{q}$.

(v) If $(\eta, X) \in J^{2,-} u_{\varepsilon}(x)$ with $x \in \Omega_{r(\varepsilon)}$, then $\eta=\frac{\left(x-x_{\varepsilon}\right)}{\varepsilon^{q-1}}\left|x_{\varepsilon}-x\right|^{q-2}$ and $X \leq \frac{q-1}{\varepsilon}|\eta|^{\frac{q-2}{q-1}} I$, where $x_{\varepsilon}$ is as in (iv).

These properties are well known, see appendix of [JJ12] and also [Kat15b] where more general "flat inf-convolution" is considered. Regardless, we give a proof of (v) based on [Kat15a, p53] due to its critical role in the proof of Lemma 5.5.

Proof of property $(v)$ in Lemma 5.2. Let $(\eta, X) \in J^{2,-} u_{\varepsilon}(x)$. Then there is a function $\varphi \in C^{2}\left(\mathbb{R}^{N}\right)$ such that it touches $u_{\varepsilon}$ from below at $x$ and $D \varphi(x)=\eta$, $D^{2} \varphi(x)=X$. Therefore for all $y, z \in \Omega$ we have

$$
u(y)+\frac{|y-z|^{q}}{q \varepsilon^{q-1}}-\varphi(z) \geq u_{\varepsilon}(z)-\varphi(z) \geq 0 .
$$


Choosing $y=x_{\varepsilon}$, we obtain

$$
\varphi(z)-\frac{\left|x_{\varepsilon}-z\right|^{q}}{q \varepsilon^{q-1}} \leq u\left(x_{\varepsilon}\right) \text { for all } z \in \Omega .
$$

Since $\varphi(x)=u_{\varepsilon}(x)=u\left(x_{\varepsilon}\right)+\frac{\left|x_{\varepsilon}-x\right|^{q}}{q \varepsilon^{q-1}}$, the above inequality means that the function

$$
z \mapsto \varphi(z)-\frac{\left|x_{\varepsilon}-z\right|^{q}}{q \varepsilon^{q-1}}=: \varphi(z)-\psi(z)
$$

has a maximum at $x$. Thus $\eta=D \psi(x)=\frac{\left(x-x_{\varepsilon}\right)}{\varepsilon^{q-1}}\left|x_{\varepsilon}-x\right|^{q-2}$ and

$$
\begin{aligned}
X \leq D^{2} \psi(x) & =\frac{1}{\varepsilon^{q-1}}\left|x_{\varepsilon}-x\right|^{q-4}\left((q-2)\left(x_{\varepsilon}-x\right) \otimes\left(x_{\varepsilon}-x\right)+\left|x_{\varepsilon}-x\right|^{2} I\right) \\
& \leq \frac{1}{\varepsilon^{q-1}}\left|x_{\varepsilon}-x\right|^{q-4}\left((q-2)\left\|\left(x_{\varepsilon}-x\right) \otimes\left(x_{\varepsilon}-x\right)\right\| I+\left|x_{\varepsilon}-x\right|^{2} I\right) \\
& =\frac{q-1}{\varepsilon^{q-1}}\left|x_{\varepsilon}-x\right|^{q-2} I \\
& =\frac{q-1}{\varepsilon^{q-1}}\left(\varepsilon|\eta|^{\frac{1}{q-1}}\right)^{q-2} I \\
& =\frac{q-1}{\varepsilon}|\eta|^{\frac{q-2}{q-1}} I .
\end{aligned}
$$

We will show that the inf-convolution provides approximations of viscosity supersolutions to $-\Delta_{p(x)}^{N} u \geq 0$. If there was no $x$-dependence in the equation, it would be straightforward to show that the inf-convolution of a supersolution is still a supersolution. However, the equation $-\Delta_{p(x)}^{N} u \geq 0$ has $x$-dependence caused by $p(x)$. Regardless, in [Ish95, Thm 3] it is shown that with some assumptions on $G$, the inf-convolution $u_{\varepsilon}$ of a viscosity supersolution to $G\left(x, u, D u, D^{2} u\right) \geq 0$ is still a viscosity supersolution to $G\left(x, u_{\varepsilon}, D u_{\varepsilon}, D^{2} u_{\varepsilon}\right) \geq E(\varepsilon)$, where $E(\varepsilon) \rightarrow 0$ as $\varepsilon \rightarrow 0$.

We prove a modified version of this theorem for the solutions of $-\Delta_{p(x)}^{N} u \geq 0$. The important modification is the term $|\eta|^{\min (p(x)-2,0)}$ in (5.2) as it cancels a singular gradient term that appears due to the error term in the proof of Lemma 5.5 , see (5.14). Another difference is that we consider inf-convolution with the exponent $q \geq 2$.

Lemma 5.3. Assume that $u$ is a uniformly continuous viscosity supersolution to $-\Delta_{p(x)}^{N} u \geq 0$ in $\Omega$. Then, whenever $(\eta, X) \in J^{2,-} u_{\varepsilon}(x), \eta \neq 0$ and $x \in \Omega_{r(\varepsilon)}$, it holds

$$
|\eta|^{\min (p(x)-2,0)} F(x, \eta, X) \geq E(\varepsilon),
$$

where $E(\varepsilon) \rightarrow 0$ as $\varepsilon \rightarrow 0$. The error function $E$ depends only on $p, q$ and the modulus of continuity of $u$.

Proof. Fix $x \in \Omega_{r(\varepsilon)}$ and $(\eta, X) \in J^{2,-} u_{\varepsilon}(x), \eta \neq 0$. Then by Lemma 5.2 there is $x_{\varepsilon} \in B_{r(\varepsilon)}(x)$ such that

$$
u_{\varepsilon}(x)=u\left(x_{\varepsilon}\right)+\frac{\left|x_{\varepsilon}-x\right|^{q}}{q \varepsilon^{q-1}}
$$

and $\eta=\frac{\left(x-x_{\varepsilon}\right)}{\varepsilon^{q-1}}\left|x_{\varepsilon}-x\right|^{q-2}$. There exists a function $\varphi \in C^{2}\left(\mathbb{R}^{N}\right)$ such that it touches $u_{\varepsilon}$ from below at $x$ and $D \varphi(x)=\eta, D^{2} \varphi(x)=X$. By the definition of inf-convolution

$$
u(y)-\varphi(z)+\frac{|y-z|^{q}}{q \varepsilon^{q-1}} \geq u_{\varepsilon}(z)-\varphi(z) \geq 0 \text { for all } y, z \in \Omega_{r(\varepsilon)} .
$$


Since by (5.3) we have $u\left(x_{\varepsilon}\right)=\varphi(x)-\frac{\left|x_{\varepsilon}-x\right|^{q}}{q \varepsilon^{q-1}}$, it follows from (5.4) that the expression $u(y)-\varphi(z)+\frac{|y-z|^{q}}{q \varepsilon^{q-1}}$ reaches its minimum at $(y, z)=\left(x_{\varepsilon}, x\right)$. Thus

$$
\max _{(y, z) \in \Omega_{r(\varepsilon)} \times \Omega_{r(\varepsilon)}}-u(y)+\varphi(z)-\frac{|y-z|^{q}}{q \varepsilon^{q-1}}=-u\left(x_{\varepsilon}\right)+\varphi(x)-\frac{\left|x_{\varepsilon}-x\right|^{q}}{q \varepsilon^{q-1}} .
$$

We denote $\Phi(y, z):=\frac{1}{q \varepsilon^{q-1}}|y-z|^{q}$ and invoke the Theorem of sums (see [CIL92]). There exist $Y, Z \in S^{N}$ such that

$$
(\eta,-Y) \in \bar{J}^{2,-} u\left(x_{\varepsilon}\right), \quad(\eta,-Z) \in \bar{J}^{2,+} \varphi(x)
$$

and

$$
\left(\begin{array}{cc}
Y & 0 \\
0 & -Z
\end{array}\right) \leq D^{2} \Phi\left(x_{\varepsilon}, x\right)+\varepsilon^{q-1}\left(D^{2} \Phi\left(x_{\varepsilon}, x\right)\right)^{2}
$$

where

$$
D^{2} \Phi\left(x_{\varepsilon}, x\right)=\left(\begin{array}{cc}
M & -M \\
-M & M
\end{array}\right)
$$

with $M=\frac{1}{\varepsilon^{q-1}}\left|x_{\varepsilon}-x\right|^{q-4}\left((q-2)\left(x_{\varepsilon}-x\right) \otimes\left(x_{\varepsilon}-x\right)+\left|x_{\varepsilon}-x\right|^{2} I\right)$ and

$$
\left(D^{2} \Phi\left(x_{\varepsilon}, x\right)\right)^{2}=2\left(\begin{array}{cc}
M^{2} & -M^{2} \\
-M^{2} & M^{2}
\end{array}\right) .
$$

The above implies $Y \leq Z \leq-D^{2} \varphi(x)=-X$. Multiplying (5.5) by the $\mathbb{R}^{2 N}$ vector $\left(\frac{\eta}{|\eta|} \sqrt{p\left(x_{\varepsilon}\right)-1}, \frac{\eta}{|\eta|} \sqrt{p(x)-1}\right)$ from both sides yields

$$
\frac{\left(p\left(x_{\varepsilon}\right)-1\right)}{|\eta|^{2}}\langle Y \eta, \eta\rangle-\frac{(p(x)-1)}{|\eta|^{2}}\langle Z \eta, \eta\rangle \leq \Lambda^{2}\left\langle\left(M+2 \varepsilon^{q-1} M^{2}\right) \frac{\eta}{|\eta|}, \frac{\eta}{|\eta|}\right\rangle,
$$

where $\Lambda=\sqrt{p(x)-1}-\sqrt{p\left(x_{\varepsilon}\right)-1}$. We have

$$
\begin{aligned}
0 \leq & F\left(x_{\varepsilon}, \eta,-Y\right) \\
= & F(x, \eta, Z)-F\left(x_{\varepsilon}, \eta, Y\right)-F(x, \eta, Z) \\
= & \left(p\left(x_{\varepsilon}\right)-1\right)\left\langle Y \frac{\eta}{|\eta|}, \frac{\eta}{|\eta|}\right\rangle-(p(x)-1)\left\langle Z \frac{\eta}{|\eta|}, \frac{\eta}{|\eta|}\right\rangle \\
& +\operatorname{tr}(Y)-\left\langle Y \frac{\eta}{|\eta|}, \frac{\eta}{|\eta|}\right\rangle-\operatorname{tr}(Z)+\left\langle Z \frac{\eta}{|\eta|}, \frac{\eta}{|\eta|}\right\rangle+F(x, \eta,-Z) \\
\leq & \Lambda^{2}\left\langle\left(M+2 \varepsilon^{q-1} M^{2}\right) \frac{\eta}{|\eta|}, \frac{\eta}{|\eta|}\right\rangle+F(x, \eta, X),
\end{aligned}
$$

where we used (5.6) and the fact that $Y \leq Z$ implies

$$
\operatorname{tr}(Y-Z)-\left\langle(Y-Z) \frac{\eta}{|\eta|}, \frac{\eta}{|\eta|}\right\rangle \leq 0
$$

We have the estimate

$$
\begin{aligned}
\|M\| & \leq \frac{1}{\varepsilon^{q-1}}\left|x_{\varepsilon}-x\right|^{q-4}\left((q-2)\left\|\left(x_{\varepsilon}-x\right) \otimes\left(x_{\varepsilon}-x\right)\right\|+\left|x_{\varepsilon}-x\right|^{2}\|I\|\right) \\
& =\frac{q-1}{\varepsilon^{q-1}}\left|x_{\varepsilon}-x\right|^{q-2} .
\end{aligned}
$$

Since $p$ is Lipschitz continuous and $p_{\min }>1$, we have also

$$
\Lambda^{2}=\frac{\left|p(x)-p\left(x_{\varepsilon}\right)\right|^{2}}{\left|\sqrt{p(x)-1}+\sqrt{p\left(x_{\varepsilon}\right)-1}\right|^{2}} \leq C(p)\left|x-x_{\varepsilon}\right|^{2} .
$$


Combining these with $(5.7)$ we get (we may assume that $r(\varepsilon)<1$ )

$$
\begin{aligned}
-F(x, \eta, X) & \leq \Lambda^{2}\left(\|M\|+2 \varepsilon^{q-1}\|M\|^{2}\right) \\
& \leq \Lambda^{2}\left(\frac{q-1}{\varepsilon^{q-1}}\left|x_{\varepsilon}-x\right|^{q-2}+2 \varepsilon^{q-1}\left(\frac{q-1}{\varepsilon^{q-1}}\right)^{2}\left|x_{\varepsilon}-x\right|^{2(q-2)}\right) \\
& \leq \frac{3(q-1)^{2}}{\varepsilon^{q-1}} \Lambda^{2}\left|x_{\varepsilon}-x\right|^{q-2} \\
& \leq C(p, q) \frac{1}{\varepsilon^{q-1}}\left|x_{\varepsilon}-x\right|^{q} .
\end{aligned}
$$

Moreover, by uniform continuity of $u$ there is a modulus of continuity $\omega$ such that $\omega(t) \rightarrow 0$ as $t \rightarrow 0$ and $|u(y)-u(z)| \leq \omega(|y-z|)$ for all $y, z \in \Omega$. Hence by (5.3)

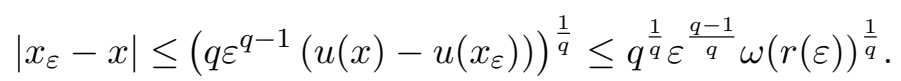

We now consider the situations $p(x) \leq 2$ and $p(x)>2$ separately.

If $p(x) \leq 2$, we multiply (5.8) by $|\eta|^{p(x)-2}$ and estimate using (5.9). We get

$$
\begin{aligned}
-|\eta|^{p(x)-2} F(x, \eta, X) & \leq C(p, q) \frac{1}{\varepsilon^{q-1}}\left|x_{\varepsilon}-x\right|^{q}|\eta|^{p(x)-2} \\
& =C(p, q) \frac{1}{\varepsilon^{q-1}}\left|x_{\varepsilon}-x\right|^{q}\left|\frac{1}{\varepsilon^{q-1}}\left(x-x_{\varepsilon}\right)\right| x_{\varepsilon}-\left.\left.x\right|^{q-2}\right|^{p(x)-2} \\
& =C(p, q)\left(\frac{1}{\varepsilon}\right)^{(q-1)(p(x)-1)}\left|x_{\varepsilon}-x\right|^{q+(q-1)(p(x)-2)} \\
& \leq C(p, q)\left(\frac{1}{\varepsilon}\right)^{(q-1)(p(x)-1)}\left(q^{\frac{1}{q} \varepsilon^{\frac{q-1}{q}}} \omega(r(\varepsilon))^{\frac{1}{q}}\right)^{q+(q-1)(p(x)-2)} \\
& =C(p, q)\left(\frac{1}{\varepsilon}\right)^{\left(\frac{q-1}{q}\right)(p(x)-2)} \omega(r(\varepsilon))^{\frac{q+(q-1)(p(x)-2)}{q}} \\
& \leq C(p, q) \omega(r(\varepsilon))^{\frac{q+(q-1)\left(p_{\min }-2\right)}{q}},
\end{aligned}
$$

where the last inequality is true when $\varepsilon<1$ is so small that $\omega(r(\varepsilon))<1$. This proves $(5.2)$ when $p(x) \leq 2$.

If $p(x)>2$, we estimate (5.8) directly using (5.9). We get

$$
\left.-F(x, \eta, X) \leq C(p, q) \frac{1}{\varepsilon^{q-1}}\left(q^{\frac{1}{q}} \varepsilon^{\frac{q-1}{q}} \omega(r(\varepsilon))^{\frac{1}{q}}\right)^{q}=C(p, q) \omega(r(\varepsilon))\right),
$$

which proves (5.2) when $p(x)>2$.

Next we will use the previous lemma to show that inf-convolution of a viscosity supersolution to $-\Delta_{p(x)}^{N} u \geq 0$ in $\Omega$ is a weak supersolution to $-\Delta_{p(x)}^{S} u \geq 0$ in $\Omega_{r(\varepsilon)}$ up to some error term. Before proceeding we make some remarks about the point-wise differentiability of inf-convolution.

Remark 5.4. It follows from semi-concavity that the inf-convolution $u_{\varepsilon}$ is locally Lipschitz in $\Omega_{r(\varepsilon)}$ (see [EG15, p267]). Therefore it belongs in $W_{l o c}^{1, \infty}\left(\Omega_{r(\varepsilon)}\right)$, is differentiable almost everywhere in $\Omega_{r(\varepsilon)}$, and its derivative agrees with its Sobolev derivative almost everywhere in $\Omega_{r(\varepsilon)}$ (see [EG15, p155 and p265]).

By Lemma 5.2 the function $\phi(x):=u_{\varepsilon}(x)-C(q, \varepsilon, u)|x|^{2}$ is concave in $\Omega_{r(\varepsilon)}$. Thus Alexandrov's theorem implies that $u_{\varepsilon}$ is twice differentiable almost everywhere in $\Omega_{r(\varepsilon)}$. Furthermore, the proof of Alexandrov's theorem in [EG15, p273] establishes that if $\phi_{j}$ is the standard mollification of $\phi$, then $D^{2} \phi_{j} \rightarrow D^{2} \phi$ almost everywhere in $\Omega_{r(\varepsilon)}$. 
Lemma 5.5. Assume that $u$ is a uniformly continuous viscosity supersolution to $-\Delta_{p(x)}^{N} u \geq 0$ in $\Omega$. Let $q>2$ be so large that $p_{\min }-2+\frac{q-2}{q-1} \geq 0$ and let $u_{\varepsilon}$ be the inf-convolution of $u$ as defined in (5.1). Then

$$
\int_{\Omega_{r(\varepsilon)}}\left|D u_{\varepsilon}\right|^{p(x)-2} D u_{\varepsilon} \cdot\left(D \varphi+\log \left|D u_{\varepsilon}\right| D p \varphi\right) d x \geq E(\varepsilon) \int_{\Omega_{r(\varepsilon)}}\left|D u_{\varepsilon}\right|^{s(x)} \varphi d x
$$

for all non-negative $\varphi \in W^{1, p(\cdot)}\left(\Omega_{r(\varepsilon)}\right)$ with compact support, where $E(\varepsilon) \rightarrow 0$ as $\varepsilon \rightarrow 0$ and $s(x)=\max (p(x)-2,0)$.

Proof. It is enough to consider $\varphi \in C_{0}^{\infty}\left(\Omega_{r(\varepsilon)}\right)$. This can be proven as Lemma 3.2, but since $u_{\varepsilon} \in W_{l o c}^{1, \infty}\left(\Omega_{r(\varepsilon)}\right)$, the proof is even simpler.

(Step 1) We show that $u_{\varepsilon}$ satisfies the auxiliary inequality (5.11) for all $0<$ $\delta<1$. As mentioned in Remark 5.4, the function $\phi(x):=u_{\varepsilon}(x)-C(q, \varepsilon, u)|x|^{2}$ is concave in $\Omega_{r(\varepsilon)}$ and therefore we can approximate it by smooth concave functions $\phi_{j}$ so that $\left(\phi_{j}, D \phi_{j}, D^{2} \phi_{j}\right) \rightarrow\left(\phi, D \phi, D^{2} \phi\right)$ almost everywhere in $\Omega_{r(\varepsilon)}$. We define

$$
u_{\varepsilon, j}(x):=\phi_{j}(x)+C(q, \varepsilon, u)|x|^{2}
$$

and denote by $p_{j}$ the standard mollification of $p$. Since $u_{\varepsilon, j}$ and $p_{j}$ are smooth, we calculate

$$
\begin{aligned}
\int_{\Omega_{r(\varepsilon)}} & -\left(\delta+\left|D u_{\varepsilon, j}\right|^{2}\right)^{\frac{p_{j}(x)-2}{2}}\left(\Delta u_{\varepsilon, j}+\frac{p_{j}(x)-2}{\delta+\left|D u_{\varepsilon, j}\right|^{2}} \Delta_{\infty} u_{\varepsilon, j}\right) \varphi d x \\
= & \int_{\Omega_{r(\varepsilon)}}-\operatorname{div}\left(\left(\delta+\left|D u_{\varepsilon, j}\right|^{2}\right)^{\frac{p_{j}(x)-2}{2}} D u_{\varepsilon, j}\right) \varphi \\
& +\frac{1}{2}\left(\delta+\left|D u_{\varepsilon, j}\right|^{2}\right)^{\frac{p_{j}(x)-2}{2}} \log \left(\delta+\left|D u_{\varepsilon, j}\right|^{2}\right) D u_{\varepsilon, j} \cdot D p_{j} \varphi d x \\
= & \int_{\Omega_{r(\varepsilon)}}\left(\delta+\left|D u_{\varepsilon, j}\right|^{2}\right)^{\frac{p_{j}(x)-2}{2}} D u_{\varepsilon, j} \cdot\left(D \varphi+\frac{1}{2} \log \left(\delta+\left|D u_{\varepsilon, j}\right|^{2}\right) D p_{j} \varphi\right) d x
\end{aligned}
$$

We let $j \rightarrow \infty$ in (5.10) and intend to use Fatou's lemma at the LHS and the Dominated convergence theorem at the RHS. This results in the auxiliary inequality

$$
\begin{aligned}
& \int_{\Omega_{r(\varepsilon)}}-\left(\delta+\left|D u_{\varepsilon}\right|^{2}\right)^{\frac{p(x)-2}{2}}\left(\Delta u_{\varepsilon}+\frac{p(x)-2}{\delta+\left|D u_{\varepsilon}\right|^{2}} \Delta_{\infty} u_{\varepsilon}\right) \varphi d x \\
& \quad \leq \int_{\Omega_{r(\varepsilon)}}\left(\delta+\left|D u_{\varepsilon}\right|^{2}\right)^{\frac{p(x)-2}{2}} D u_{\varepsilon} \cdot\left(D \varphi+\frac{1}{2} \log \left(\delta+\left|D u_{\varepsilon}\right|^{2}\right) D p \varphi\right) d x
\end{aligned}
$$

where $D^{2} u_{\varepsilon}$ is the Hessian of $u_{\varepsilon}$ in the Alexandrov's sense. We still need to check that the assumptions of the Dominated convergence theorem and Fatou's lemma hold. By Lipschitz continuity of $u_{\varepsilon}$ and $p$ there is $M \geq 1$ such that

$$
\sup _{j}\left\|D u_{\varepsilon, j}\right\|_{L^{\infty}(\operatorname{supp} \varphi)}, \sup _{j}\left\|D p_{j}\right\|_{L^{\infty}(\operatorname{supp} \varphi)} \leq M .
$$

This justifies our use of the Dominated convergence theorem. In order to justify our use of Fatou's lemma, we notice first that by concavity of $\phi_{j}$ we have $D^{2} u_{\varepsilon, j} \leq$ $C(q, \varepsilon, u) I$. Thus the integrand at the LHS of (5.10) is clearly bounded from below 
by a constant independent of $j$ if $D u_{\varepsilon, j}=0$. If $D u_{\varepsilon, j} \neq 0$, we have

$$
\begin{aligned}
(\delta & \left.+\left|D u_{\varepsilon, j}\right|^{2}\right)^{\frac{p_{j}(x)-2}{2}}\left(\Delta u_{\varepsilon, j}+\frac{p_{j}(x)-2}{\delta+\left|D u_{\varepsilon, j}\right|^{2}} \Delta_{\infty} u_{\varepsilon, j}\right) \\
& =\frac{\left(\delta+\left|D u_{\varepsilon, j}\right|^{2}\right)^{\frac{p_{j}(x)-2}{2}}}{\delta+\left|D u_{\varepsilon, j}\right|^{2}}\left(\left|D u_{\varepsilon, j}\right|^{2}\left(\Delta u_{\varepsilon, j}+\frac{p_{j}(x)-2}{\left|D u_{\varepsilon, j}\right|^{2}} \Delta_{\infty} u_{\varepsilon, j}\right)+\delta \Delta u_{\varepsilon, j}\right) \\
& \leq \frac{\delta^{\frac{p_{j}(x)-2}{2}}+\left(\delta+M^{2}\right)^{\frac{p_{j}(x)-2}{2}}}{\delta+\left|D u_{\varepsilon, j}\right|^{2}} C(q, \varepsilon, u)\left(\left|D u_{\varepsilon, j}\right|^{2}\left(N+p_{j}(x)-2\right)+\delta N\right) \\
& \leq C(q, \varepsilon, u)\left(\delta^{\frac{p_{\min }-2}{2}}+\left(\delta+M^{2}\right)^{\frac{p_{\max }-2}{2}}\right)\left(2 N+p_{\max }-2\right),
\end{aligned}
$$

where the first inequality follows like estimate (5.7) since $p_{j} \geq p_{\min }>1$.

(Step 2) We let $\delta \rightarrow 0$ in the auxiliary inequality (5.11). The RHS becomes

$$
\int_{\Omega_{r(\varepsilon)} \backslash\left\{D u_{\varepsilon}=0\right\}}\left|D u_{\varepsilon}\right|^{p(x)-2} D u_{\varepsilon} \cdot\left(D \varphi+\log \left|D u_{\varepsilon}\right| D p \varphi\right) d x
$$

by the Lebesgue's dominated convergence theorem. We intend to apply Fatou's lemma on the LHS. We have $\left(D u_{\varepsilon}(x), D^{2} u_{\varepsilon}(x)\right) \in J^{2,-} u_{\varepsilon}(x)$ for almost every $x \in$ $\Omega_{r(\varepsilon)}$. Therefore by Lemma 5.3 it holds that

$$
\left|D u_{\varepsilon}\right|^{\min (p(x)-2,0)} F\left(x, D u_{\varepsilon}, D^{2} u_{\varepsilon}\right) \geq E(\varepsilon) \text { in }\left\{x \in \Omega_{r(\varepsilon)}: D u_{\varepsilon} \neq 0\right\}
$$

and by the property (v) in Lemma 5.2 we have

$$
D^{2} u_{\varepsilon} \leq \frac{q-1}{\varepsilon}\left|D u_{\varepsilon}\right|^{\frac{q-2}{q-1}} I
$$

Observe that since $q>2$, the condition (5.13) implies that the Hessian $D^{2} u_{\varepsilon}$ is negative semi-definite in the set where the gradient $D u_{\varepsilon}$ vanishes. Using this fact, Fatou's lemma and (5.12) we get

$$
\begin{aligned}
\liminf _{\delta \rightarrow 0} & \int_{\Omega_{r(\varepsilon)}}-\left(\left|D u_{\varepsilon}\right|^{2}+\delta\right)^{\frac{p(x)-2}{2}}\left(\Delta u_{\varepsilon}+\frac{p(x)-2}{\left|D u_{\varepsilon}\right|^{2}+\delta} \Delta_{\infty} u_{\varepsilon}\right) \varphi d x \\
\geq & \liminf _{\delta \rightarrow 0} \int_{\left\{D u_{\varepsilon} \neq 0\right\}}-\left(\left|D u_{\varepsilon}\right|^{2}+\delta\right)^{\frac{p(x)-2}{2}}\left(\Delta u_{\varepsilon}+\frac{p(x)-2}{\left|D u_{\varepsilon}\right|^{2}+\delta} \Delta_{\infty} u_{\varepsilon}\right) \varphi d x \\
& \quad+\liminf _{\delta \rightarrow 0} \int_{\left\{D u_{\varepsilon}=0\right\}}-\delta^{\frac{p(x)-2}{2}} \Delta u_{\varepsilon} \varphi d x \\
\geq & \int_{\left\{D u_{\varepsilon} \neq 0\right\}}-\left|D u_{\varepsilon}\right|^{p(x)-2}\left(\Delta u_{\varepsilon}+\frac{p(x)-2}{\left|D u_{\varepsilon}\right|^{2}} \Delta_{\infty} u_{\varepsilon}\right) \varphi d x \\
\geq & E(\varepsilon) \int_{\left\{D u_{\varepsilon} \neq 0\right\}}\left|D u_{\varepsilon}\right|^{\max (p(x)-2,0)} \varphi d x
\end{aligned}
$$


and thus we arrive at the desired inequality. Our use of Fatou's lemma is justified since if $D u_{\varepsilon} \neq 0$ and $p(x) \leq 2$, we have by $(5.13)$

$$
\begin{aligned}
\left(\left|D u_{\varepsilon}\right|^{2}+\delta\right)^{\frac{p(x)-2}{2}}\left(\Delta u_{\varepsilon}+\frac{p(x)-2}{\left|D u_{\varepsilon}\right|^{2}+\delta} \Delta_{\infty} u_{\varepsilon}\right) \\
\quad=\frac{\left(\left|D u_{\varepsilon}\right|^{2}+\delta\right)^{\frac{p(x)-2}{2}}}{\left|D u_{\varepsilon}\right|^{2}+\delta}\left(\left|D u_{\varepsilon}\right|^{2}\left(\Delta u_{\varepsilon}+\frac{p(x)-2}{\left|D u_{\varepsilon}\right|^{2}} \Delta_{\infty} u_{\varepsilon}\right)+\delta \Delta u_{\varepsilon}\right) \\
\quad \leq \frac{\left(\left|D u_{\varepsilon}\right|^{2}+\delta\right)^{\frac{p(x)-2}{2}}}{\left|D u_{\varepsilon}\right|^{2}+\delta} \frac{q-1}{\varepsilon}\left(\left|D u_{\varepsilon}\right|^{\frac{q-2}{q-1}+2}(N+p(x)-2)+\left|D u_{\varepsilon}\right|^{\frac{q-2}{q-1}} \delta N\right) \\
\leq\left|D u_{\varepsilon}\right|^{p(x)-2+\frac{q-2}{q-1}}\left(\frac{q-1}{\varepsilon}\right)(2 N+p(x)-2) \\
\leq\left(\left\|D u_{\varepsilon}\right\|_{L^{\infty}(\operatorname{supp} \varphi)}+1\right)^{p_{\max }-2+\frac{q-2}{q-1}}\left(\frac{q-1}{\varepsilon}\right)\left(2 N+p_{\max }-2\right)
\end{aligned}
$$

where the last inequality follows from $p_{\min }-2+\frac{q-2}{q-1} \geq 0$. If $D u_{\varepsilon} \neq 0$ and $p(x)>2$, we have

$$
\begin{aligned}
& \left(\left|D u_{\varepsilon}\right|^{2}+\delta\right)^{\frac{p(x)-2}{2}}\left(\Delta u_{\varepsilon}+\frac{p(x)-2}{\left|D u_{\varepsilon}\right|^{2}+\delta} \Delta_{\infty} u_{\varepsilon}\right) \\
& \quad \leq\left(\left\|D u_{\varepsilon}\right\|_{L^{\infty}(\operatorname{supp} \varphi)}^{2}+1\right)^{\frac{p_{\max }-2}{2}+\frac{q-2}{q-1}}\left(\frac{q-1}{\varepsilon}\right)\left(N+p_{\max }-2\right)
\end{aligned}
$$

In the next two lemmas we use Caccioppoli type estimates and algebraic inequalities to show that the sequence of inf-convolutions converges to the viscosity supersolution in $W_{l o c}^{1, p(\cdot)}(\Omega)$.

Lemma 5.6. Under the assumptions of Lemma 5.5, the function $u$ belongs in $W_{\text {loc }}^{1, p(\cdot)}(\Omega)$ and for any $\Omega^{\prime} \Subset \Omega$ we have $D u_{\varepsilon} \rightarrow D u$ weakly in $L^{p(\cdot)}\left(\Omega^{\prime}\right)$ for some subsequence.

Proof. Take a cut-off function $\xi \in C_{0}^{\infty}\left(\Omega^{\prime}\right)$ such that $0 \leq \xi \leq 1$ in $\Omega$ and $\xi \equiv 1$ in $\Omega^{\prime}$. Then assume that $\varepsilon$ is so small that $\operatorname{supp} \xi=: K \subset \Omega_{r(\varepsilon)}$. We define a test function $\varphi:=\left(L-u_{\varepsilon}\right) \xi^{p_{\max }}$ where $L:=\sup _{\varepsilon, x \in \Omega^{\prime}}\left|u_{\varepsilon}(x)\right|$ is finite since $u_{\varepsilon} \rightarrow u$ locally uniformly. We have

$$
D \varphi=-D u_{\varepsilon} \xi^{p_{\max }}+\left(L-u_{\varepsilon}\right) p^{+} \xi^{p_{\max }-1} D \xi
$$

and therefore by Lemma 5.5

$$
\begin{aligned}
\int_{\Omega_{r(\varepsilon)}}\left|D u_{\varepsilon}\right|^{p(x)} \xi^{p_{\max }} d x \leq & \int_{\Omega_{r(\varepsilon)}}\left|D u_{\varepsilon}\right|^{p(x)-1} \xi^{p_{\max }-1}\left(L-u_{\varepsilon}\right) p_{\max }|D \xi| d x \\
& +\int_{\Omega_{r(\varepsilon)}}\left|D u_{\varepsilon}\right|^{p(x)-1}|\log | D u_{\varepsilon}|||D p|\left(L-u_{\varepsilon}\right) \xi^{p_{\max }} d x \\
& +|E(\varepsilon)| \int_{\Omega_{r(\varepsilon)}}\left|D u_{\varepsilon}\right|^{\max (p(x)-2,0)}\left(L-u_{\varepsilon}\right) \xi^{p_{\max }} d x \\
= & : I_{1}+I_{2}+I_{3} .
\end{aligned}
$$


We estimate these integrals using Young's inequality. The first integral is estimated by the facts $\frac{p(x)\left(p_{\max }-1\right)}{p(x)-1} \geq p_{\max }$ and $\xi \leq 1$ as follows

$$
\begin{aligned}
I_{1} & \leq \int_{\Omega_{r(\varepsilon)}} \delta\left|D u_{\varepsilon}\right|^{p(x)} \xi^{\frac{p(x)\left(p_{\max }-1\right)}{p(x)-1}}+\left(\frac{2}{\delta} L p_{\max }|D \xi|\right)^{p(x)} d x \\
& \leq \delta \int_{\Omega_{r(\varepsilon)}}\left|D u_{\varepsilon}\right|^{p(x)} \xi^{p_{\max }} d x+C(\delta, p, L, D \xi) .
\end{aligned}
$$

To estimate $I_{2}$, we also use the inequality $a^{s}|\log a| \leq a^{s+\frac{1}{2}}+\frac{1}{s}$ for $a>0$ and $s>0$,

$$
\begin{aligned}
I_{2} & \leq \int_{\Omega_{r(\varepsilon)}}\left(\left|D u_{\varepsilon}\right|^{p(x)-\frac{1}{2}}+\frac{1}{p(x)-1}\right) \xi^{p_{\max }}|D p| 2 L d x \\
& \leq \int_{\Omega_{r(\varepsilon)}} \delta\left|D u_{\varepsilon}\right|^{p(x)} \xi^{\frac{p_{\max p(x)}(x)-\frac{1}{2}}{p}}+\left(\frac{2}{\delta}|D p| L\right)^{2 p(x)}+\frac{2 L|D p| \xi^{p_{\max }}}{p_{\min }-1} d x \\
& \leq \delta \int_{\Omega_{r(\varepsilon)}}\left|D u_{\varepsilon}\right|^{p(x)} \xi^{p_{\max }} d x+C(\delta, p, D p, L) .
\end{aligned}
$$

The last integral is estimated by the two alternatives in $\max (p(x)-2,0)$ as follows (we may assume that $|E(\varepsilon)| \leq 1$ )

$$
\begin{aligned}
I_{3} & \leq \int_{\Omega_{r(\varepsilon)} \cap\{p(x)>2\}}\left|D u_{\varepsilon}\right|^{p(x)-2} \xi^{p_{\max }} 2 L d x+\int_{\Omega_{r(\varepsilon)} \cap\{p(x) \leq 2\}} 2 L \xi^{p_{\max }} d x \\
& \leq \int_{\Omega_{r(\varepsilon)} \cap\{p(x)>2\}} \delta\left|D u_{\varepsilon}\right|^{p(x)} \xi^{\frac{p_{\max }(x)}{p(x)-2}}+\left(\frac{2}{\delta} L\right)^{\frac{p(x)}{2}} d x+C(p, L) \\
& \leq \delta \int_{\Omega_{r(\varepsilon)}}\left|D u_{\varepsilon}\right|^{p(x)} \xi^{p_{\max }} d x+C(\delta, p, L) .
\end{aligned}
$$

Taking small $\delta$ we conclude that $D u_{\varepsilon}$ is bounded in $L^{p(\cdot)}\left(\Omega^{\prime}\right)$ with respect to $\varepsilon$. Since $L^{p(\cdot)}\left(\Omega^{\prime}\right)$ is a reflexive Banach space [DHHR11, p76 and p89], it follows that there is a function $D u \in L^{p(\cdot)}\left(\Omega^{\prime}\right)$ such that $D u_{\varepsilon} \rightarrow D u$ weakly in $L^{p(\cdot)}\left(\Omega^{\prime}\right)$ for some subsequence. Consequently $u \in W^{1, p(\cdot)}\left(\Omega^{\prime}\right)$ with $D u$ as its weak derivative.

Lemma 5.7. Under the assumptions of Lemma 5.5, for any $\Omega^{\prime} \Subset \Omega$ we have $D u_{\varepsilon} \rightarrow D u$ in $L^{p(\cdot)}\left(\Omega^{\prime}\right)$ for some subsequence.

Proof. Take a cut-off function $\xi \in C_{0}^{\infty}(\Omega)$ such that $\xi \equiv 1$ in $\Omega^{\prime}$ and define a test function $\varphi:=\left(u-u_{\varepsilon}\right) \xi$. Then assume that $\varepsilon$ is so small that $\operatorname{supp} \xi=: K \subset \Omega_{r(\varepsilon)}$. 
Since $\varphi \in W^{1, p(\cdot)}\left(\Omega_{r(\varepsilon)}\right)$ with compact support it follows from Lemma 5.5 that

$$
\begin{aligned}
\int_{\Omega_{r(\varepsilon)}} & \left(|D u|^{p(x)-2} D u-\left|D u_{\varepsilon}\right|^{p(x)-2} D u_{\varepsilon}\right) \cdot\left(D u-D u_{\varepsilon}\right) \xi d x \\
\leq & \int_{\Omega_{r(\varepsilon)}}\left|D u_{\varepsilon}\right|^{p(x)-2} D u_{\varepsilon} \cdot D \xi\left(u-u_{\varepsilon}\right) d x \\
& +\int_{\Omega_{r(\varepsilon)}}\left|D u_{\varepsilon}\right|^{p(x)-2} \log \left(\left|D u_{\varepsilon}\right|\right) D u_{\varepsilon} \cdot D p\left(u-u_{\varepsilon}\right) \xi d x \\
& +|E(\varepsilon)| \int_{\Omega_{r(\varepsilon)}}\left|D u_{\varepsilon}\right|^{\max (p(x)-2,0)}\left(u-u_{\varepsilon}\right) \xi d x \\
& +\int_{\Omega_{r(\varepsilon)}}|D u|^{p(x)-2} D u \cdot\left(D u-D u_{\varepsilon}\right) \xi d x \\
\leq & \left\|u-u_{\varepsilon}\right\|_{L^{\infty}(K)} \int_{K}\left(C\left(p_{\min }\right)+\left|D u_{\varepsilon}\right|^{p(x)}\right)(D \xi+|D p|+|E(\varepsilon)|) d x \\
& +\int_{K}|D u|^{p(x)-2} D u \cdot\left(D u-D u_{\varepsilon}\right) \xi d x .
\end{aligned}
$$

According to Lemma 5.6 we have $u_{\varepsilon} \rightarrow u$ locally uniformly and $D u_{\varepsilon} \rightarrow D u$ weakly in $L^{p(\cdot)}(K)$ for a subsequence. Thus by passing to a subsequence we may assume that the right hand side of (5.15) converges to zero. The claim now follows from the inequalities (see e.g. [Lin17, Chapter 12])

$$
\begin{aligned}
\left(|a|^{p(x)-2} a\right. & \left.-|b|^{p(x)-2} b\right) \cdot(a-b) \\
& \geq \begin{cases}(p(x)-1)|a-b|^{2}\left(1+|a|^{2}+|b|^{2}\right)^{\frac{p(x)-2}{2}} & p(x)<2 \\
2^{2-p(x)}|a-b|^{p(x)} & p(x) \geq 2\end{cases}
\end{aligned}
$$

for $a, b \in \mathbb{R}^{N}$. Indeed, we immediately get that $\int_{\Omega^{\prime} \cap\{p(x) \geq 2\}}\left|D u-D u_{\varepsilon}\right|^{p(x)} d x \rightarrow$ 0 . To deal with the set $\{p(x)<2\}$, we first apply the above algebraic inequality and then estimate using Hölder's inequality, the modular inequality (2.1) and the definition of the $\|\cdot\|_{L^{p(\cdot)}}$-norm. We get

$$
\begin{aligned}
& \int_{\Omega^{\prime} \cap\{p(x)<2\}}\left|D u-D u_{\varepsilon}\right|^{p(x)} d x \\
& \leq \int_{\Omega^{\prime} \cap\{p(x)<2\}}\left(\left(|D u|^{p(x)-2} D u-\left|D u_{\varepsilon}\right|^{p(x)-2} D u_{\varepsilon}\right) \cdot\left(D u-D u_{\varepsilon}\right)\right)^{\frac{p(x)}{2}} \\
& \quad \cdot\left(\frac{1}{p(x)-1}\right)^{\frac{p(x)}{2}}\left(1+|D u|^{2}+\left|D u_{\varepsilon}\right|^{2}\right)^{\frac{p(x)(2-p(x))}{4}} d x \\
& \leq\left\|\left(\left(|D u|^{p(x)-2} D u-\left|D u_{\varepsilon}\right|^{p(x)-2} D u_{\varepsilon}\right) \cdot\left(D u-D u_{\varepsilon}\right)\right)^{\frac{p(x)}{2}}\right\|_{L^{\frac{2}{p(\cdot)}}\left(\Omega^{\prime} \cap\{p(x)<2\}\right)} \\
& \quad \cdot \frac{2}{p_{\min }-1}\left\|\left(1+|D u|^{2}+\left|D u_{\varepsilon}\right|^{2}\right)^{\frac{p(x)(2-p(x))}{4}}\right\| \\
& \leq\left(\int_{\Omega_{r(\varepsilon)}}\left(|D u|^{p(x)-2} D u-\left|D u_{\varepsilon}\right|^{p(x)-2} D u_{\varepsilon}\right) \cdot\left(D u-D u_{\varepsilon}\right) \xi d x\right)^{s}\left(\Omega^{\prime} \cap\{p(x)<2\}\right) \\
& \cdot \frac{2}{p_{\min }-1}\left(1+\int_{\Omega^{\prime} \cap\{p(x)<2\}}\left(1+|D u|^{2}+\left|D u_{\varepsilon}\right|^{2}\right)^{\frac{p(x)}{2}} d x\right)
\end{aligned}
$$


where $s \in\left\{\frac{p_{\max }}{2}, \frac{p_{\min }}{2}\right\}$. The last integral is bounded since the sequence $D u_{\varepsilon}$ is bounded in $L^{p(\cdot)}\left(\Omega^{\prime}\right)$ by its weak convergence. The RHS therefore converges to zero by (5.15).

Next, we use the previous convergence result to pass to the limit in the inequality of Lemma 5.5 and conclude that viscosity supersolutions to $-\Delta_{p(x)}^{N} u \geq 0$ are weak supersolutions to $-\Delta_{p(x)}^{S} u \geq 0$.

Theorem 5.8. If $u \in C(\Omega)$ is a viscosity supersolution to $-\Delta_{p(x)}^{N} u \geq 0$ in $\Omega$, then $u$ is a weak supersolution to $-\Delta_{p(x)}^{S} u \geq 0$ in $\Omega$.

Proof. It is clear from the definition of weak supersolutions to $-\Delta_{p(x)}^{S} u \geq 0$ that we can without loss of generality assume that $u$ is uniformly continuous in $\Omega$ by restricting to a smaller domain. Fix a non-negative test function $\varphi \in C_{0}^{\infty}(\Omega)$ and take an open $\Omega^{\prime} \Subset \Omega$ such that $\operatorname{supp} \varphi \subset \Omega^{\prime}$. Let $q$ and $u_{\varepsilon}$ be as in Lemma 5.5 and assume that $\varepsilon$ is so small that $\Omega^{\prime} \subset \Omega_{r(\varepsilon)}$. Then the claim follows from Lemma 5.5 if we show that

$$
\lim _{\varepsilon \rightarrow 0} \int_{\Omega^{\prime}}\left|D u_{\varepsilon}\right|^{p(x)-2} D u_{\varepsilon} \cdot D \varphi d x=\int_{\Omega^{\prime}}|D u|^{p(x)-2} D u \cdot D \varphi d x
$$

and

$$
\begin{gathered}
\lim _{\varepsilon \rightarrow 0} \int_{\Omega^{\prime}}\left|D u_{\varepsilon}\right|^{p(x)-2} \log \left(\left|D u_{\varepsilon}\right|\right) D u_{\varepsilon} \cdot D p \varphi d x \\
=\int_{\Omega^{\prime}}|D u|^{p(x)-2} \log (|D u|) D u \cdot D p \varphi d x
\end{gathered}
$$

as well as

$$
\lim _{\varepsilon \rightarrow 0} E(\varepsilon) \int_{\Omega^{\prime}}\left|D u_{\varepsilon}\right|^{\max (p(x)-2,0)} \varphi d x=0 .
$$

By Lemma 5.7 we have that $u_{\varepsilon} \rightarrow u$ in $W^{1, p(\cdot)}\left(\Omega^{\prime}\right)$.

Claim (5.16) follows from the inequalities (see e.g. [Lin17, Chapter 12])

$$
\left.|| a\right|^{p(x)-2} a-|b|^{p(x)-2} b \mid \leq \begin{cases}2^{2-p(x)}|a-b|^{p(x)-1} & p(x)<2 \\ 2^{-1}\left(|a|^{p(x)-2}+|b|^{p(x)-2}\right)|a-b| & p(x) \geq 2\end{cases}
$$

for $a, b \in \mathbb{R}^{N}$. Indeed, when $\varepsilon$ is so small that $\int_{\Omega^{\prime}}\left|D u_{\varepsilon}-D u\right|^{p(x)} d x<1$ we have by Hölder's inequality and the modular inequality

$$
\begin{aligned}
& \left.\int_{\Omega^{\prime}}|| D u_{\varepsilon}\right|^{p(x)-2} D u_{\varepsilon}-|D u|^{p(x)-2} D u \mid d x \\
& \leq 2 \int_{\Omega^{\prime} \cap\{p(x)<2\}}\left|D u_{\varepsilon}-D u\right|^{p(x)-1} d x \\
& \quad+2^{-1} \int_{\Omega^{\prime} \cap\{p(x) \geq 2\}}\left(\left|D u_{\varepsilon}\right|^{p(x)-2}+|D u|^{p(x)-2}\right)\left|D u_{\varepsilon}-D u\right| d x \\
& \leq C(p, \Omega)\left(\int_{\Omega^{\prime}}\left|D u_{\varepsilon}-D u\right|^{p(x)} d x\right)^{\frac{1}{p_{\max }}} \\
& \quad+C(p, \Omega)\left(1+\int_{\Omega^{\prime}}\left|D u_{\varepsilon}\right|^{p(x)}+|D u|^{p(x)} d x\right)\left\|D u_{\varepsilon}-D u\right\|_{L^{p(\cdot)}\left(\Omega^{\prime}\right)}
\end{aligned}
$$

Claim (5.18) holds since $\int_{\Omega^{\prime}}\left|D u_{\varepsilon}\right|^{p(x)} d x$ is bounded and $E(\varepsilon) \rightarrow 0$.

Claim (5.17) follows if we show that

$$
\left.\lim _{\varepsilon \rightarrow 0} \int_{\Omega^{\prime}}|| D u_{\varepsilon}\right|^{p(x)-2} \log \left(\left|D u_{\varepsilon}\right|\right) D u_{\varepsilon}-|D u|^{p(x)-2} \log (|D u|) D u \mid d x=0 .
$$


To this end, fix $0<\epsilon<1$. The mapping $(a, x) \mapsto|a|^{p(x)-2} \log (|a|) a$ is uniformly continuous in bounded sets of $\mathbb{R}^{N} \times \Omega^{\prime}$. Hence there exists $\delta=\delta(\epsilon)<\epsilon$ such that whenever $x \in \Omega^{\prime}$ and $a, b \in \bar{B}(0,3)$ satisfy $|a-b|<\delta$, it holds

$$
\left.|| a\right|^{p(x)-2} \log (|a|) a-|b|^{p(x)-2} \log (|b|) b \mid \leq \epsilon .
$$

If $|a|,|b| \geq 1$ and $|a-b|<\delta$, then we use (5.19) to get the estimate

$$
\begin{aligned}
& \left.|| a\right|^{p(x)-2} \log (|a|) a-|b|^{p(x)-2} \log (|b|) b \mid \\
& \leq|b|^{p(x)-1}|\log | a|-\log | b||+\left.|\log | a|||| a\right|^{p(x)-2} a-|b|^{p(x)-2} b \mid \\
& \leq|b|^{p(x)}|a-b|+|a| \cdot \begin{cases}2^{2-p(x)}|a-b|^{p(x)-1}, & p(x)<2 \\
2^{-1}\left(|a|^{p(x)-2}+|b|^{p(x)-2}\right)|a-b|, \quad p(x) \geq 2\end{cases} \\
& \leq\left(1+2^{-1}\right)\left(|a|^{p(x)}+|b|^{p(x)}\right)|a-b|+2|a||a-b|^{p(x)-1} \\
& \leq C\left(|a|^{p(x)}+|b|^{p(x)}\right) \epsilon^{\min \left(p_{\min }-1,1\right)}
\end{aligned}
$$

We denote

$$
F_{\varepsilon}=\left\{x \in \Omega^{\prime}:\left|D u_{\varepsilon}(x)-D u(x)\right| \geq \delta\right\} .
$$

The strong convergence of $D u_{\varepsilon}$ to $D u$ in $L^{p(\cdot)}\left(\Omega^{\prime}\right)$ implies that $D u_{\varepsilon} \rightarrow D u$ in measure in $\Omega^{\prime}$ (see [DHHR11, Lemma 3.2.10]). Thus there is $\varepsilon_{0}=\varepsilon_{0}(\delta)$ such that for all $\varepsilon<\varepsilon_{0}$ it holds $\left|F_{\varepsilon}\right| \leq \delta$. Using the inequality $a^{s}|\log a| \leq a^{s+\frac{1}{2}}+\frac{1}{s}$ for $a, s>0$, we get for all $\varepsilon<\varepsilon_{0}$

$$
\begin{aligned}
& \left.\int_{F_{\varepsilon}}|| D u_{\varepsilon}\right|^{p(x)-2} \log \left(\left|D u_{\varepsilon}\right|\right) D u_{\varepsilon}-|D u|^{p(x)-2} \log (|D u|) D u \mid d x \\
& \leq \int_{F_{\varepsilon}} \frac{2}{p(x)-1}+\left|D u_{\varepsilon}\right|^{p(x)-\frac{1}{2}}+|D u|^{p(x)-\frac{1}{2}} d x \\
& \leq C\left(p_{\min }\right)\left|F_{\varepsilon}\right|+\|1\|_{L^{2 p(\cdot)}\left(F_{\varepsilon}\right)}\left(\left\|D u_{\varepsilon}\right\| \frac{p(\cdot)}{L^{p(\cdot)-\frac{1}{2}}\left(F_{\varepsilon}\right)}+\|D u\| \frac{p(\cdot)}{L^{p(\cdot)-\frac{1}{2}}\left(F_{\varepsilon}\right)}\right) \\
& \leq C\left(p_{\min }\right)\left|F_{\varepsilon}\right|+\mid F_{\varepsilon} \frac{1}{\frac{1}{\max }^{2 p_{\max }}}\left(2+\int_{F_{\varepsilon}}\left|D u_{\varepsilon}\right|^{p(x)}+|D u|^{p(x)} d x\right) \\
& \leq C\left(p_{\min }\right)\left(1+\int_{\Omega^{\prime}}\left|D u_{\varepsilon}\right|^{p(x)}+|D u|^{p(x)} d x\right) \epsilon^{\frac{1}{2 p_{\max }}} .
\end{aligned}
$$

If $x \in \Omega^{\prime} \backslash F_{\varepsilon}$, then either $\left|D u_{\varepsilon}\right|,|D u| \leq 3$ or $\left|D u_{\varepsilon}\right|,|D u| \geq 1$. Hence by (5.21) and (5.22) we have

$$
\begin{aligned}
& \left.\int_{\Omega^{\prime} \backslash F_{\varepsilon}}|| D u_{\varepsilon}\right|^{p(x)-2} \log \left(\left|D u_{\varepsilon}\right|\right) D u_{\varepsilon}-|D u|^{p(x)-2} \log (|D u|) D u \mid d x \\
& \leq C\left(\int_{\Omega^{\prime}}\left|D u_{\varepsilon}\right|^{p(x)}+|D u|^{p(x)}+1 d x\right) \epsilon^{\min \left(p_{\min }-1,1\right)} .
\end{aligned}
$$

Combining (5.24) and (5.23) proves (5.20) since $\epsilon$ was arbitrary.

Merging Theorems 4.1 and 5.8 yields the following equivalence result.

Theorem 5.9. A function $u$ is a viscosity solution to $-\Delta_{p(x)}^{N} u=0$ in $\Omega$ if and only if it is a weak solution to $-\Delta_{p(x)}^{S} u=0$ in $\Omega$.

Since the weak solutions to the strong $p(x)$-Laplace equation are locally $C^{1, \alpha}$ continuous [ZZ12], our equivalence result yields local $C^{1, \alpha}$ regularity also for viscosity solutions of the normalized $p(x)$-Laplace equation. 
Corollary 5.10. If $u$ is a viscosity solution to $-\Delta_{p(x)}^{N} u=0$ in a bounded domain $\Omega$, then $u \in C^{1, \alpha}(\Omega)$ with $\alpha \in(0,1)$.

\section{An Applichtion: A Radó-Type Removability theOREM}

The classical theorem of Radó says that if a continuous complex-valued function $f$ defined on a domain $\Omega \subset \mathbb{C}$ is holomorphic in $\Omega \backslash\{f=0\}$, then it is holomorphic in the whole $\Omega$. Similar results have been proven for solutions of partial differential equations. We prove a Radó-type removability theorem for the strong $p(x)$-Laplace equation. It is worth pointing out that it could be difficult to show this kind of result without appealing to viscosity solutions whereas it is straightforward to do so with the help of the equivalence result. The theorem follows by observing that weak solutions to $\Delta_{p(x)}^{S} u=0$ coincide with viscosity solutions of an equation that satisfies the assumptions of a Radó-type removability theorem in [JL05].

Recall that we ignore the test functions whose gradient vanishes at the point of touching in the Definition 3.3 of viscosity solutions to $-\Delta_{p(x)}^{N} u=0$. Sometimes this kind of solutions are called feeble viscosity solutions (e.g. [JL05, Kat15b]). We will observe that these feeble viscosity solutions to $-\Delta_{p(x)}^{N} u=0$ are exactly the usual viscosity solutions to

$$
-\operatorname{tr}\left(A(x, D u) D^{2} u\right)=0,
$$

where $A(x, D u):=|D u|^{2} I+(p(x)-2) D u \otimes D u$. To be precise, we define the viscosity solutions to $(6.1)$.

Definition 6.1. A lower semicontinuous function $u$ is a viscosity supersolution to (6.1) in $\Omega$ if, whenever $(\eta, X) \in J^{2,-} u(x)$ with $x \in \Omega$, then

$$
-\operatorname{tr}(A(x, \eta) X) \geq 0 \text {. }
$$

A function $u$ is a viscosity subsolution to (6.1) if $-u$ is a supersolution, and a viscosity solution if it is both viscosity super- and subsolution.

Lemma 6.2. A function $u$ is a viscosity solution to $-\Delta_{p(x)}^{N} u=0$ if and only if it is a viscosity solution to (6.1).

Proof. It is enough to consider supersolutions. Take $(\eta, X) \in J^{2,-} u(x)$ with $x \in \Omega$. If $\eta=0$, then the conditions for both definitions are satisfied, so we may assume that $\eta \neq 0$. Then we have

if and only if

$$
F(x, \eta, X) \geq 0
$$

where

$$
-\left(|\eta|^{2} \operatorname{tr}(X)+(p(x)-2)\langle X \eta, \eta\rangle\right) \geq 0
$$

$$
\begin{aligned}
|\eta|^{2} \operatorname{tr}(X)+(p(x)-2)\langle X \eta, \eta\rangle & =|\eta|^{2} \operatorname{tr}(X)+(p(x)-2) \operatorname{tr}(\eta \otimes \eta X) \\
& =\operatorname{tr}\left(\left(|\eta|^{2} I+(p(x)-2) \eta \otimes \eta\right) X\right)
\end{aligned}
$$

Hence the definitions are equivalent.

Theorem 6.3 (A Radó-type removability theorem). Let $u \in C^{1}(\Omega)$ be a weak solution to $-\Delta_{p(x)}^{S} u=0$ in $\Omega \backslash\{u=0\}$. Then $u$ is a weak solution to $-\Delta_{p(x)}^{S} u=0$ in the whole $\Omega$.

Proof. By Lemma 6.2 and our equivalence result weak solutions to $-\Delta_{p(x)}^{S} u=0$ coincide with viscosity solutions to (6.1). Therefore it suffices to show that if $u$ is a viscosity solution to (6.1) in $\Omega \backslash\{u=0\}$, it is a viscosity solution to (6.1) in the whole $\Omega$. This on the other hand follows from [JL05, Theorem 2.2]. The matrix $A$ satisfies the assumptions of the theorem as it is symmetric, has continuous entries 
and $A(x, 0,0)=0$ for all $x \in \Omega$. It is also positive semi-definite since for all $\xi \in \mathbb{R}^{N}$ we have

$$
\begin{aligned}
\xi^{\prime}\left(|\eta|^{2} I+(p(x)-2) \eta \otimes \eta\right) \xi & \geq \xi^{\prime}\left(|\eta|^{2} I-\eta \otimes \eta\right) \xi \\
& \geq|\xi|^{2}\left(|\eta|^{2}-\|\eta \otimes \eta\|\right)=0
\end{aligned}
$$

\section{REFERENCES}

[AH10] T. Adamowicz and P. Hästö. Mappings of finite distortion and PDE with nonstandard growth. Int. Math. Res. Not. IMRN, 10:1940-1965, 2010.

[AH11] T. Adamowicz and P. Hästö. Harnack's inequality and the strong $p(\cdot)$-Laplacian. $J$. Differential Equations, 250:1631-1649, 2011.

[AHP17] Á. Arroyo, J. Heino, and M. Parviainen. Tug-of-war games with varying probabilities and the normalized $p(x)$-Laplacian. Commun. Pure Appl. Anal., 16(3):915-944, 2017.

[APR17] A. Attouchi, M. Parviainen, and E. Ruosteenoja. $C^{1, \alpha}$ regularity for the normalized p-Poisson problem. J. Math. Pures Appl., 108(4):553-591, 2017.

[BG15] A. Banerjee and N. Garofalo. Modica type gradient estimates for an inhomogeneus variant of the normalized $p$-Laplacian evolution. Nonlinear Anal., 121:458-468, 2015.

[CIL92] M. G. Crandall, H. Ishii, and P.-L. Lions. User's guide to viscosity solutions of second order partial differential equations. Bull. Amer. Math. Soc., 27(1):1-67, 1992.

[DHHR11] L. Diening, P. Harjulehto, P. Hästö, and M. Růžička. Lebesgue and Sobolev Spaces with Variable Exponents, volume 2017 of Lecture Notes in Mathematics. SpringerVerlag, 2011.

[EG15] L. C. Evans and R. F. Gariepy. Measure Theory and Fine Properties of Functions. CRC Press, revised edition, 2015.

[IJS] C. Imbert, T. Jin, and L. Silvestre. Hölder gradient estimates for a class of singular or degenerate parabolic equations. To appear in Adv. Nonlinear Anal.

[Ish95] H. Ishii. On the equivalence of two notions of weak solutions, viscosity solutions and distribution solutions. Funkcialaj Ekvacioj, 38:101-120, 1995.

[JJ12] V. Julin and P. Juutinen. A new proof for the equivalence of weak and viscosity solutions for the $p$-Laplace equation. Comm. Partial Differential Equations, 37(5):934946, 2012.

[JL05] P. Juutinen and P. Lindqvist. Removability of a level set for solutions of quasilinear equations. Comm. Partial Differential Equations, 30:305-321, 2005.

[JLM01] P. Juutinen, P. Lindqvist, and J.J. Manfredi. On the equivalence of viscosity solutions and weak solutions for a quasi-linear equation. SIAM J. Math. Anal., 33(3):699-717, 2001.

[JLP10] P. Juutinen, T. Lukkari, and M. Parviainen. Equivalence of viscosity and weak solutions for the $p(x)$-Laplacian. Ann. Inst. H. Poincaré Anal. Non Linéaire, 27(6):1471$1487,2010$.

[JS17] T. Jin and L. Silvestre. Hölder gradient estimates for parabolic homogeneous p-Laplacian equations. J. Math. Pures Appl., 108(3):63-87, 2017.

[Kat15a] N. Katzourakis. An Introduction To Viscosity Solutions for Fully Nonlinear PDE with Applications to Calculus of Variations in $L^{\infty}$. Springer, 2015.

[Kat15b] N. Katzourakis. Nonsmooth convex functionals and feeble viscosity solutions of singular Euler-Lagrange equations. Calc. Var., 54(1):275-298, 2015.

[Koi12] Shigeaki Koike. A Beginner's Guide to the Theory of Viscosity Solutions. 2nd edition, 2012.

[Lin17] P. Lindqvist. Notes on the p-Laplace equation (second edition). Univ. Jyväskylä, Report 161, 2017.

[MO] M. Medina and P. Ochoa. On viscosity and weak solutions for non-homogeneous $p$-Laplace equations. To appear in Adv. Nonlinear Anal.

[MPR12] J. J. Manfredi, M. Parviainen, and J.D. Rossi. On the definition and properties of p-harmonous functions. Ann. Sc. Norm. Super. Pisa Cl. Sci. (5), 11(2):215-241, 2012.

[PL13] M. Pérez-Llanos. A homogenization process for the strong $p(x)$-Laplacian. Nonlinear Anal., 76:105-114, 2013.

[PS08] Y. Peres and S. Sheffield. Tug-of-war with noise: A game-theoretic view of the p-Laplacian. Duke Math. J., 145(1):91-120, 2008.

[PSSW09] Y. Peres, O. Schramm, S. Sheffield, and D.B. Wilson. Tug-of-war and the infinity Laplacian. J. Amer. Math. Soc., 22(1):167-210, 2009. 
[ZZ12] C. Zhang and S. Zhou. Hölder regularity for the gradients of solutions of the strong p(x)-Laplacian. J. Math. Anal. Appl., 389(2):1066-1077, 2012.

[ZZZ17] C. Zhang, X. Zhang, and S. Zhou. Gradient estimates for the strong $p(x)$-Laplace equation. Discrete Contin. Dyn. Syst., 37(7):4109-4129, 2017.

Jarkko Siltakoski, Department of Mathematics and Statistics, P.O.Box 35, Fin-40014, University of Jyväskylä, Finland

E-mail address: jarkko.j.m.siltakoski@student.jyu.fi 